\title{
PDMS AND SILICON MICROMECHANISMS IN A MONOLITHIC PROCESS
}

\author{
A. P. Gerratt ${ }^{*}$, I. Penskiy ${ }^{l}$, and S. Bergbreiter ${ }^{1,2}$ \\ ${ }^{1}$ Department of Mechanical Engineering, University of Maryland, College Park, USA \\ ${ }^{2}$ Institute for Systems Research, University of Maryland, College Park, USA
}

\begin{abstract}
A new PDMS and silicon fabrication process that integrates a soft elastomer in-plane with silicon features has been developed, characterized and demonstrated. The simple three-mask process uses deep reactive ion etching of silicon trenches on a silicon-oninsulator wafer to pattern high aspect ratio silicon and elastomer features from $2 \mu \mathrm{m}$ to hundreds of micrometers in size. The process has been characterized with Sylgard 184 and is demonstrated in compliant hinges that can be operated both in- and out-of-plane and in a micromechanical thruster. These components demonstrate a new versatility in MEMS processing that allows for simple fabrication of robust features that can undergo large deformations.
\end{abstract}

\section{INTRODUCTION}

The most universal materials used in microfabrication include semiconductors, metals, and dielectrics, all materials with Young's moduli on the order of GPa's [1]. Softer materials are common in microfluidic applications and are typically fabricated with soft lithography [2-3]. The material most commonly used for soft lithography is poly(dimethylsiloxane) (PDMS). The advantages of PDMS over typical microfabrication materials are robustness, ability to withstand strains in excess of $100 \%$ without plastic deformation, and a low Young's modulus. While there are a few examples of systems that have integrated typical silicon bulk and surface micromachining process with PDMS processing [4-5], these devices requires complex fabrication that would be difficult to adapt for other applications.

The purpose of this research was to develop a process that enables integration of PDMS into a standard silicon-on-insulator (SOI) fabrication process for manufacturing monolithic micromechanisms. This process has to be repeatable, have a high yield, and use batch fabrication techniques (require no manual assembly).

Two devices that exemplify the value of integrating PDMS and silicon in a monolithic process are compliant hinges and a micromechanical elastomer-based thruster. A hinge with a compliant joint would allow for more robust manipulation both inand out-of-plane using smaller forces compared to a similar device fabricated with traditional MEMS materials. A micromechanical thruster with an embedded elastomer would allow for repeatedly storing and quickly releasing energy with an elastomer spring in a monolithic device. Smaller forces are required to store the energy in elastomer compared to similar silicon devices [6].

\section{FABRICATION PROCESS}

The fabrication process for PDMS and silicon micromechanisms is shown in Figure 1. The process is very similar to the commercially available SOI-MUMPS process with the addition of an extra mask step to define the PDMS features [7]. Devices were all fabricated on a silicon-on-insulator (SOI) wafer (430 $\mu \mathrm{m}$ handle layer thickness, $2 \mu \mathrm{m}$ buried oxide (BOX) layer thickness, and $20 \mu \mathrm{m}$ device layer thickness). In step 1, photoresist was patterned on the device layer and a deep reactive ion etch (DRIE) was performed down to the buried oxide layer.

Sylgard 184 PDMS from Dow Corning was mixed in a 10:1 ratio of base to curing agent and degassed in vacuum at 1 Torr for 15 minutes. In step 2, the PDMS was spread across the surface, degassed again, and cured.

The PDMS was planarized to the surface of the wafer in step 3. This was accomplished by using a razor blade as a squeegee to scrape the wafer surface. The remaining after step 1 photoresist was used to liftoff any residual PDMS. The liftoff leaves small particles of PDMS on the surface of the wafer, so a brief soak in nmethylpyrrolidone (NMP) and tetrabutylammonium fluoride (TBAF) in a 3:1 ratio was performed [2].

In step 4, photoresist was again patterned on the device layer of the wafer and another DRIE was performed down to the buried oxide. Photoresist was then spun on the top of the wafer to protect it during later processing steps.

In step 5, photoresist was patterned on the backside of the wafer, after which the device layer of the wafer was bonded with a thin layer of photoresist to another handle wafer. A DRIE was performed on the backside of the SOI wafer down to the buried
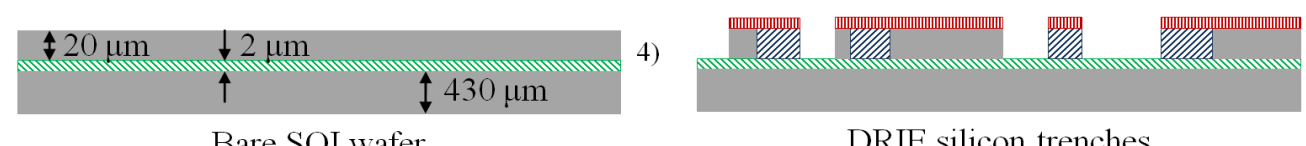

Bare SOI wafer

1)

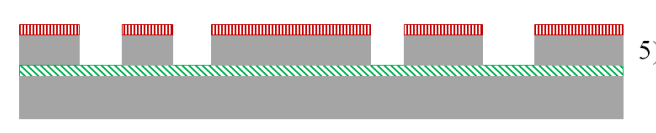

DRIE elastomer trenches

2)

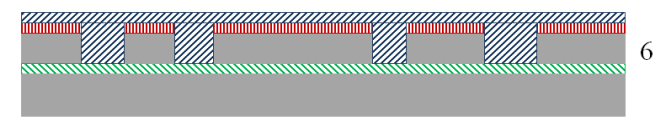

Refill trenches with elastomer

3)

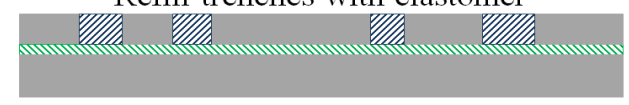

Planarize elastomer to silicon surface

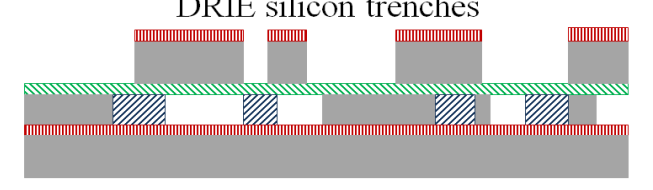

DRIE on backside

6)
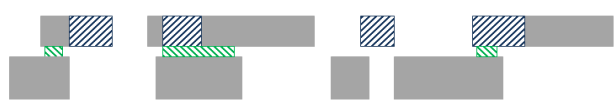

Release of buried oxide

Figure 1: Microfabrication Process 


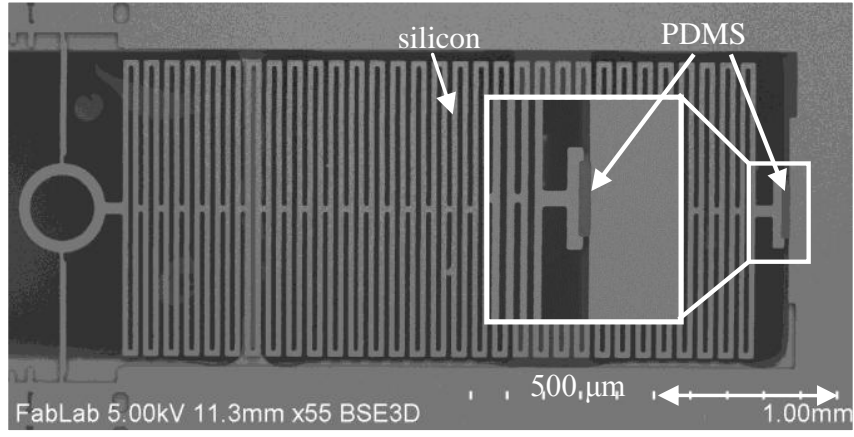

Figure 2: SEM of the test structure used to measure normal adhesion

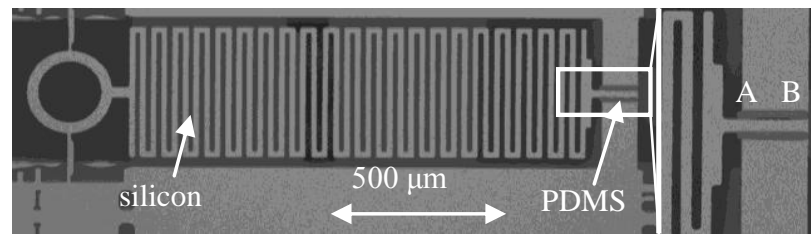

Figure 3: SEM of the test structure used to measure shear adhesion

oxide layer. A solvent release was used to separate the SOI wafer from the handle wafer. Finally in step 6 , an 18:1 buffered hydrofluoric acid (BHF) etch of the buried oxide layer was used to release some of the silicon and PDMS features.

\section{PROCESS CHARACTERIZATION}

The microfabrication process described above was developed to enable the manufacture of PDMS and silicon features in an SOI wafer. The unique challenges for this process are refilling high aspect ratio trenches with viscous elastomers, survival of PDMS features during release etch, sufficient adhesion between the silicon and PDMS so that the two materials do not delaminate, and comparable mechanical properties between unprocessed and processed PDMS, particularly the ultimate strain and Young's modulus.

\section{Feature size and Aspect ratio}

High aspect ratio trenches in excess of 20:1 have been successfully refilled with several different elastomers. The viscosities of the implemented pre-cure elastomers range from 4,575 centipoise to 66,700 centipoise [8]. Features widths from 2 $\mu \mathrm{m}$ to hundreds of micrometers have been fabricated at lengths up to several millimeters. The yield for features less than about $30 \mu \mathrm{m}$ in width was near $100 \%$. As the width of the features increases above $30 \mu \mathrm{m}$, the yield decreases. Any variations in the surface topology around the elastomer features will result in a local thinning of the photoresist, especially at wider features. This is problematic for SOI wafers with thick device layers as the photoresist masking layer is not always sufficient around the elastomer features to withstand the entire DRIE in step 4. This issue can be mitigated by using a thicker masking layer or a masking material with a superior selectivity with silicon, such as silicon dioxide.

\section{Silicon-Elastomer Adhesion}

Adhesion of the silicon and elastomer features is crucial for proper operation of the fabricated devices. Ideal adhesion would allow the elastomer to reach its ultimate strain before the silicon and elastomer delaminate. Test structures to examine the shear

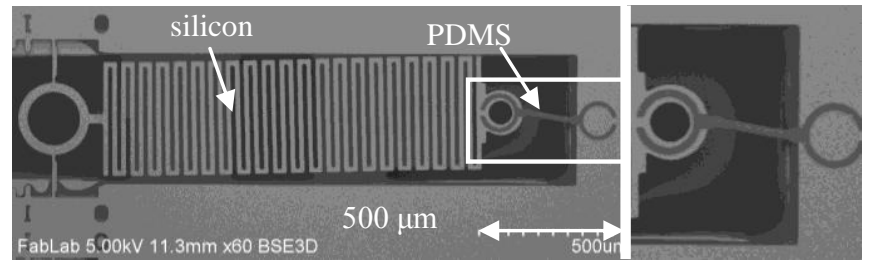

Figure 4: SEM images of the test structure used to measure stressstrain and the Young's modulus

adhesion and normal adhesion have been designed and fabricated. These structures consist of the elastomer that is under investigation in series with a silicon spring. The silicon spring was designed using ANSYS to have a spring constant of $4.35 \mathrm{~N} / \mathrm{m}$. The spring constant was recalculated after fabrication to account for any lateral etch during the DRIE. A probe was used to manually apply a force to the end of the silicon spring and the same force was transferred to the elastomer. For analysis, micrographs are taken at several stages as the applied force was increased.

The test structure used to measure the normal adhesion is shown in Figure 2. The ultimate pressure at which the elastomer delaminated from the silicon due to a normal force occurred over the range of $0.8 \mathrm{MPa}$ to $1.6 \mathrm{MPa}$ in eight tests. The test structure used to measure the shear adhesion is shown in Figure 3. The ultimate pressure at which the elastomer delaminated from the silicon due to a shear force occurred over the range of 40 to $70 \mathrm{kPa}$ in five tests, which is significantly lower than the normal condition.

In the normal force condition, the force is well distributed across the width of the elastomer, resulting in a constant pressure across the elastomer. In the shear force condition, the force is not well distributed along the length of the elastomer and the section closest to the silicon spring (point $\mathrm{A}$ in Figure 3) experiences a larger stress than the elastomer section farthest from the silicon spring (point B in Figure 3). This stress concentration leads to a premature delamination of the elastomer from the silicon that progresses along the length of the elastomer from point A to point B. For future designs, adhesion can be maximized by increasing the surface area of the silicon and elastomer interface and eliminating any stress concentrations.

\section{Stress-Strain Behavior}

The ultimate strain and stress-strain behavior of the elastomer is important for understanding the mechanics of any device, especially one which may use the elastomer for mechanical energy storage or for hinges that will undergo large strains. The test structure used to determine these behaviors is shown in Figure 4. The structure has a silicon spring in series with a long elastomer spring. The tests brought the structures to failure, which was typically due to delamination of the elastomer at an average elongation of $116 \%$ over eight tests, but was twice due to failure of the elastomer spring. Elongation of both the elastomer spring and the silicon spring are used to calculate the response of the elastomer. The measured stress-strain curves of Sylgard 184 for seven separate test structures are shown in Figure 5. The initial linear region of the curve was used to calculate an average Young's modulus of $1.4 \mathrm{MPa}$ for the Sylgard 184. This is in close agreement with the reported modulus for unprocessed Sylgard 184 at the macro-scale [9]. It is important to note that at strains above about $75 \%$, the response becomes non-linear, so the measured 1.4 MPa Young's modulus is only valid below that threshold.

While the failure mechanism for most structures was delamination from the silicon surface, strains as high as about 


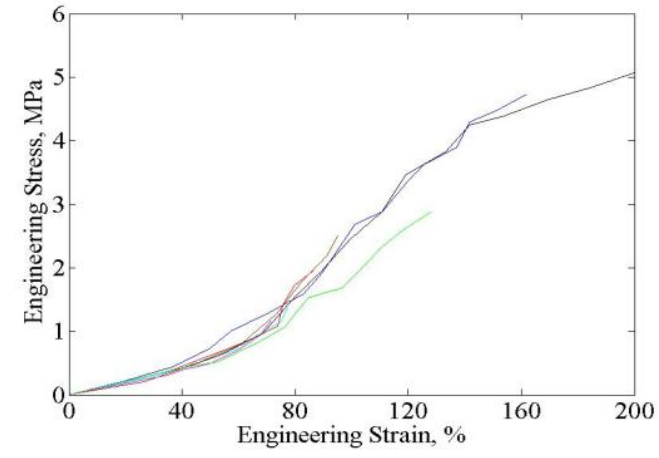

Figure 5: Eight stress-strain curves for Sylgard 184

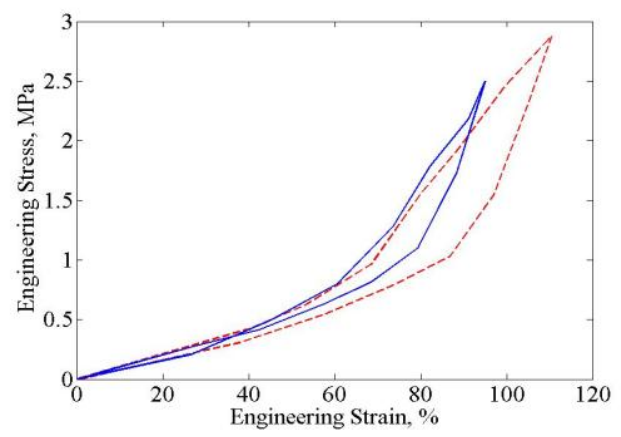

Figure 6: Two hysteresis curves for Sylgard 184

$200 \%$ have been achieved, which is slightly greater than the $180 \%$ maximum strain that has been reported in literature for unprocessed Sylgard 184 [9].

\section{Hysteresis}

Stretching and relaxing an elastomer incurs losses due to heating and friction from the rubbing of the crosslinked polymer chains as they reorganize during elongation or contraction of the bulk [10]. Two tests of the hysteresis for fabricated Sylgard 184 are shown in Figure 6. The solid blue curve had 15\% energy losses in the relaxation of the Sylgard 184 and the dashed red curve had $27 \%$ energy losses in the relaxation at a higher maximum strain. These tests were performed by slowly straining and relaxing the elastomer spring, as opposed to the rapid release that will be demonstrated later. Prior work showed negligible hysteresis in unprocessed Sylgard 184 [11], so it is possible that additional process development can mitigate the energy loss.

\section{RESULTS}

The PDMS and silicon process presented here has been used to fabricate both micromechanical thrusters and hinges. Both of these micromechanisms have enhanced performance over prior works, which are discussed.

\section{Micromechanical Thrusters}

One application of this process is in the storage and quick release of mechanical energy in a microthruster. In prior work, Rodgers demonstrated storage and release of $19 \mathrm{~nJ}$ of energy in a surface micromachined device that used electrostatic comb drive actuators to store energy in bending of polysilicon flexures [6]. This device, however, could only be operated once as it used a fuse-like mechanism that had to be blown in order to release the stored energy. Bergbreiter demonstrated the storage and release of $1.2 \mu \mathrm{J}$ of energy in an elastomer spring with a process that used separately fabricated and manually assembled silicon and

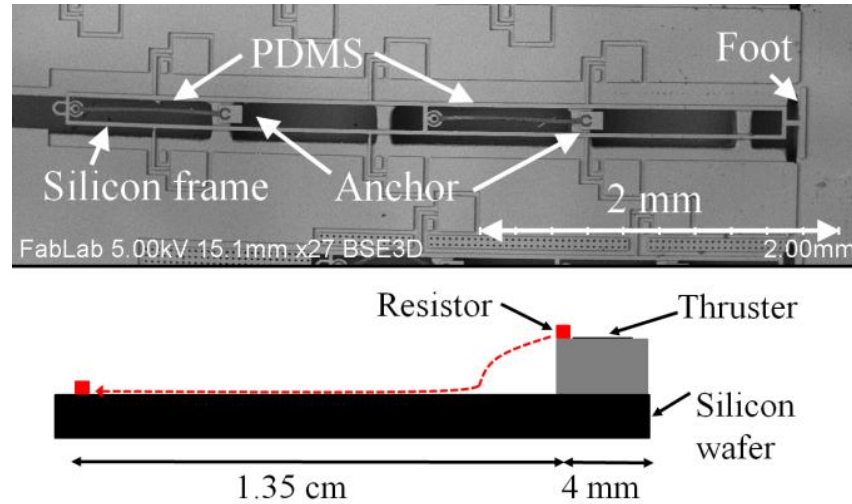

Figure 7: Micromechanical silicon and elastomer thruster and a schematic showing the thruster setup

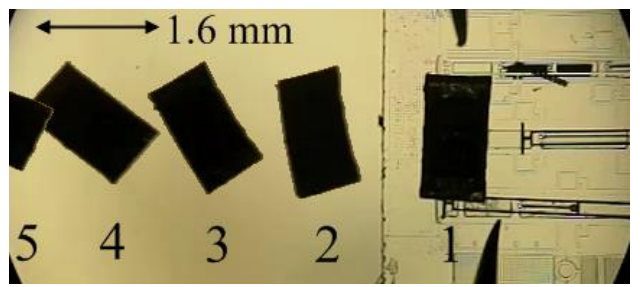

Figure 8: Superimposed screenshots of a thruster kicking a $2 \mathrm{mg}$ resistor

elastomer components, resulting in a serial process [12].

In this work, a reusable monolithic thruster capable of storing and releasing $1.3 \mu \mathrm{J}$ to propel a $2 \mathrm{mg}$ projectile $1.35 \mathrm{~cm}$ has been demonstrated. A full description of this device was presented in [13] and is summarized here. The thruster, shown in Figure 7, has two elastomer springs that are anchored at one end and attached to a silicon frame at the other end. The silicon frame surrounds the springs and has a foot at the front end that can be used to kick projectiles.

The fabricated thruster in Figure 7 was used to propel a $2 \mathrm{mg}$ $1.6 \mathrm{~mm}$ by $0.8 \mathrm{~mm}$ by $0.45 \mathrm{~mm}$ surface mount resistor in order to demonstrate the quick energy release capabilities of the elastomer. A probe was used to pull the frame of the thruster back, straining the two Sylgard 184 springs. The springs were stretched about 1 $\mathrm{mm}$, or to $130 \%$ strain. The energy stored in the springs was estimated at $1.3 \mu \mathrm{J}$. If the hysteretic losses are conservatively estimated to be $30 \%$, this corresponds to the release of $0.9 \mu \mathrm{J}$.

The probe was then removed, releasing the frame which then kicked the resistor. Five frames from a video of the resistor moving from right to left are shown in Figure $8 ; 1$ is at $0 \mathrm{~ms}, 2$ is at $8 \mathrm{~ms}, 3$ is at $12 \mathrm{~ms}, 4$ is at $17 \mathrm{~ms}$, and 5 is at $23 \mathrm{~ms}$. The remaining trajectory of the resistor is unknown since it was outside the view of the microscope, but the resistor travelled a measured distance of $1.35 \mathrm{~cm}$. The release took $5 \mathrm{~ms}$, and assuming $0.9 \mu \mathrm{J}$ was released, the elastomer has a power output of $233 \mathrm{~mW} / \mathrm{mm}^{3}$. The same thruster was used to propel the resistor several times, demonstrating repeatable actuation.

\section{Micromechanical Hinges}

Microfabricated hinges and joints typically use thin polysilicon staples to hold silicon or polysilicon pins in place [14]. In this section, compliant, high yield, and robust hinges are demonstrated that integrate a silicon beam with a compliant PDMS joint, as shown in Figure 9. The hinges shown here allow for rotation both in- and out-of-plane. Silicon cantilevers are connected to the wafer substrate with small sections of elastomer. 


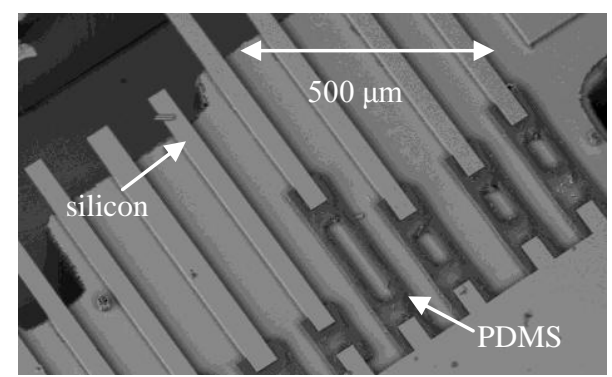

Figure 9: SEM image of silicon and PDMS hinges

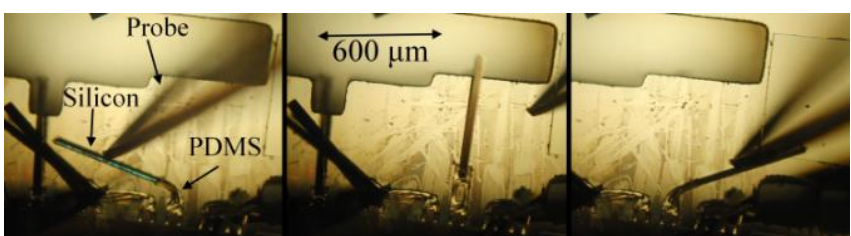

Figure 10: Manipulation of a silicon cantilever with a probe inplane

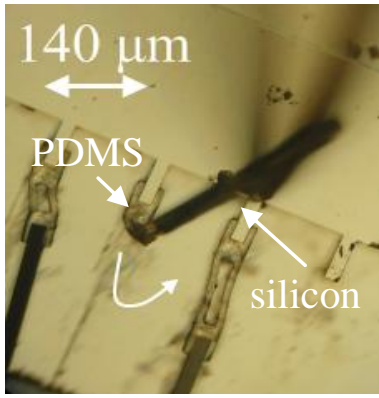

Figure 11: Manipulation of a silicon

cantilever with a probe out-of-plane

A close-up SEM of several hinges is shown in Figure 9. These cantilevers can be easily rotated both in- and out-of-plane, after release, with a probe tip. Testing of manipulation in-plane is demonstrated in Figure 10 and testing out-of-plane is demonstrated in Figure 11. The cantilever is shown in three positions, pushed $90^{\circ}$ to the left with a probe, as fabricated, and pushed $90^{\circ}$ to the right with a probe. This cantilever was rotated in-plane $180^{\circ}$ over 200 times and up out-of-plane $90^{\circ}$ over 500 times with no visual signs of wear. When it was rotated side-to-side, the cantilever would also occasionally twist about $90^{\circ}$ along its length. While these are initial demonstrations of hinges, optimized hinges can provide robust and compact replacements of polysilicon hinges.

\section{CONCLUSIONS AND FUTURE WORK}

This paper describes the fabrication, characterization, and demonstration of monolithic PDMS and silicon micromechanisms. The mechanical properties of the post-processed elastomer were studied and a modulus of 1.4 MPa was measured. The process was demonstrated in fabrication of a micromechanical thruster that was used to store and release about $1 \mu \mathrm{J}$ of energy to propel a $2 \mathrm{mg}$ resistor $1.35 \mathrm{~cm}$, with an energy density of $233 \mathrm{~mW} / \mathrm{mm}^{3}$. The process was also used to demonstrate compliant hinges. These hinges are an improvement over previous attempts at MEMSfabricated hinges [14] because of their compliance and their ability to be manipulated both in- and out-of-plane.

Future work will expand this process to new materials, such as the MRTV-1 (another soft elastomer), which can withstand many hundreds of percent strain with no plastic deformation, and more rigid materials such as SU-8 or parylene. Lifetime tests need to be performed and new elastomers still need to be characterized for further application of the process. The energy release system will also be integrated with electrostatic inchworm motors in order to demonstrate an autonomous jumping silicon chip with embedded elastomer.

\section{ACKNOWLEDGEMENTS}

The authors gratefully acknowledge the support of the Maryland NanoCenter and its FabLab. This work was supported under DARPA YFA Grant No. HR0011-08-1-0043.

\section{REFERENCES}

[1] K.E. Peterson, "Silicon as a Mechanical Material," Proceedings of the IEEE, 70, 1982, 420-457.

[2] J.N. Lee, C. Park, and G.M. Whitesides, "Solvent compatibility of poly(dimethylsiloxane)-based microfluidic devices," Analytical Chemistry, 75, 2003, 6544-6554.

[3] D.C. Duffy, J.C. McDonald, O.J.A. Schueller, and G.M. Whitesides, "Rapid Prototyping of Microfluidic Systems in Poly(dimethylsiloxane)," Analytical Chemistry, 70, 1998, 4974-4984.

[4] Y. Tung and K. Kurabayashi, "A single-layer PDMS-onsilicon hybrid microactuator with multi-axis out-of-plane motion capabilities-Part II: Fabrication and Characterization," Journal of Microelectromechanical Systems, 14, 2005, 558-566.

[5] J.C. Lotters, W. Olthuis, P.H. Veltink, and P. Bergveld, "Design, realization and characterization of a symmetrical triaxial capacitive accelerometer for medical applications," Sensors and Actuators A: Physical, 61, 1997, 303-308.

[6] M.S. Rodgers, J.J. Allen, K.D. Meeks, B.D. Jensen, and S.L. Miller, "A micromechanical high-density energy storage / rapid release system," SPIE Conference on Micromachined Devices and Components V, Santa Clara, CA: 1999, 212--222.

[7] "SOIMUMPS," http://www.memscap.com/, 2008.

[8] "Information about Dow Corning Brand Silicone Encapsulants," http://www2.dowcorning.com/DataFiles/090007c88020bcc a.pdf, 2010.

[9] F. Schneider, T. Fellner, J. Wilde, and U. Wallrabe, "Mechanical properties of silicones for MEMS," Journal of Micromechanics and Microengineering, 18, 2008, 065008.

[10] Y.P. Khanna, E.A. Turi, T.J. Taylor, V.V. Vickroy, and R.F. Abbott, "Dynamic mechanical relaxations in polyethylene," Macromolecules, 18, 1985, 1302-1309.

[11] K.L. Mills, X. Zhu, S. Takayama, and M.D. Thouless, "The mechanical properties of a surface-modified layer on poly (dimethylsiloxane)," Journal of materials research, 23, 2008, 37.

[12] S. Bergbreiter, D. Mahajan, and K.S.J. Pister, "A reusable micromechanical energy storage/quick release system with assembled elastomers," Journal of Micromechanics and Microengineering, 19, 2009, 055009.

[13] A.P. Gerratt, I. Penskiy, and S. Bergbreiter, "High Power Micromechanical Thrusters with Embedded Elastomer," Proceedings of PowerMEMS 2009, Washington, DC, Dec. 1-4 2009.

[14] K.S.J. Pister, M.W. Judy, and others, "Microfabricated hinges," Sensors and Actuators A, 33, 1992, 249-256.

\section{CONTACT}

*A. P. Gerratt; gerratt@umd.edu 